\title{
The Stability Balloon for Two-dimensional Vortex Ripple Patterns
}

\author{
J. L. Hansen ${ }^{1,2}$, M. van Hecke ${ }^{1}$, C. Ellegaard ${ }^{1}$, K. H. Andersen ${ }^{1,3}$, T. Bohr ${ }^{4}$, A. Haaning ${ }^{1}$ and T. Sams ${ }^{2}$ \\ ${ }^{1}$ Niels Bohr Institute, Blegdamsvej 17, DK-2100 Copenhagen Ø \\ ${ }^{2}$ Danish Defense Research Establishment, Ryvangs allé 1, Postbox 2715, DK-2100 Copenhagen Ø \\ 3 ISVA, Danish Technical University, building 115, DK-2800 Kgs. Lyngby \\ ${ }^{4}$ Institute of Physics, Danish Technical University, Building 309, DK-2800 Kgs. Lyngby
}

(October 30, 2018)

\begin{abstract}
Patterns of vortex ripples form when a sand bed is subjected to an oscillatory fluid flow. Here we describe experiments on the response of regular vortex ripple patterns to sudden changes of the driving amplitude $a$ or frequency $f$. A sufficient decrease of $f$ leads to a "freezing" of the pattern, while a sufficient increase of $f$ leads to a supercritical secondary "pearling" instability. Sufficient changes in the amplitude $a$ lead to subcritical secondary "doubling" and "bulging" instabilities. Our findings are summarized in a "stability balloon" for vortex ripple pattern formation.
\end{abstract}

47.54.+r, 45.70.Qj, 47.20.Lz,

A flat bed of sand subjected to an oscillatory flow of water is seldom stable but instead displays the formation of patterns. Classical studies [1] have shown that after the flat bed becomes linearly unstable, so-called rolling grain ripples (small heaps of grains) are formed first. These are, however, always transient [2], and eventually strongly nonlinear vortex ripples are formed in a coarsening type process. These ripples have triangular crests with slopes roughly at the angle of repose, and the flow around the ripple crests is dominated by vortices that occur in the wake of the ripples. Together with the converging flow at the "wind" side of the ripples, these vortices yield sand mass transport directed toward the crests of the ripples, which is balanced by sand avalanching down when the slopes grow too large. The wavelength of such ripple patterns is comparable to the amplitude of the fluid motion which sets the scale for the size of the separation bubbles [2 4]; this wavelength is substantially larger than the most unstable wavelength of a flat bed [5, 6.

To characterize the vortex ripples we have studied their pattern forming properties. From this perspective, the system combines a number of unique features. Firstly, the driving is anisotropic which results in alignment of the ripples perpendicular to the flow. This allows for studies in one-dimensional geometries [2,8], although our experiments indicate that instabilities of the ripples lead to intrinsically two dimensional patterns. Secondly, typical ripple wavelengths are essentially independent of the system and grain dimensions and $f$, but scale with the driving amplitude $a$ [2]3]. Finally, due to the strongly nonlinear character of the development, it has so far not been possible to describe the pattern dynamics in terms of an "amplitude equation".

Our setup consists of a tray of sand that is oscillated with amplitude $a$ and frequency $f$ in a tank of water, allowing us to study two-dimensional patterns. To probe the fully developed vortex ripples, we have studied their response to sudden changes of the control parameters $a$

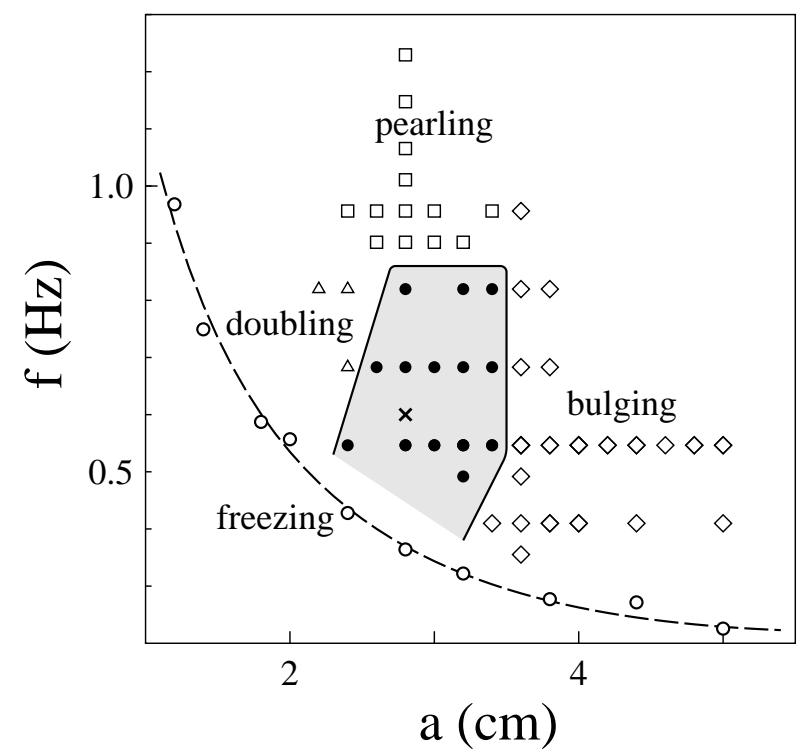

FIG. 1. Stability balloon for fully developed vortex ripples. The initial ripple pattern with wavelength $4.2 \mathrm{~cm}$ is compatible with driving parameters $a=2.8 \mathrm{~cm}$ and $f=0.6 \mathrm{~Hz}$ (cross) and remains stable for drivings indicated in full circles. Triangles, squares and diamonds indicate drivings where this pattern experiences a doubling, pearling or bulging instability. The open circles correspond to the $n=10$ measurements shown in Fig. 3 and the dashed line roughly indicates where the pattern freezes.

and $f$. Our findings lead to a "stability balloon" for vortex ripple pattern formation shown in Fig. 11. First we study the amount of grain motion for patterns that have evolved "freely" from the flat bed and have a wavelength selected by the driving amplitude. This leads to the "freezing line" in Fig. 1 (and discussed in more detail in Fig. 3). In the second set of experiments we study the response of regular patterns with initially fixed wavelengths to changes in $f$ or $a$. We find that a secondary "pearling" instability (Fig. 4) occurs when the frequency is sufficiently increased, while secondary "doubling" 


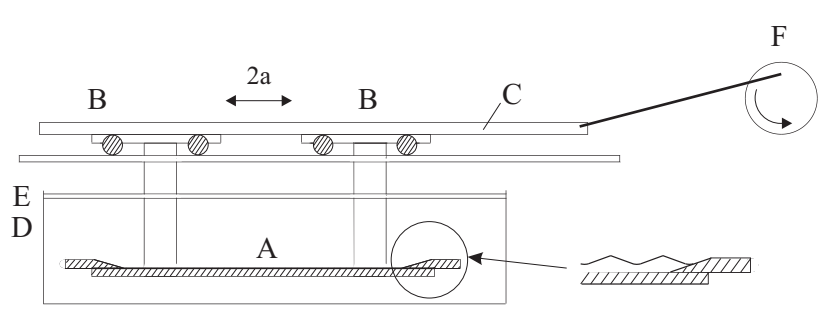

FIG. 2. Sketch of the experimental setup. The tray A is filled with sand and its oscillations are driven by the motor $\mathrm{F}$ via the rail $\mathrm{B}$.

and "bulging" (Fig. 5) instabilities occur when the amplitude $a$ is decreased or increased respectively, beyond some threshold. A qualitative presentation of these instabilities has appeared in [9].

Experimental Setup - Our setup is sketched in Fig. 2. An $0.6 \mathrm{~m} \times 1 \mathrm{~m}$ aluminum tray (A) suspended from rollers (B) mounted on a stiff frame (C) is immersed in a sufficiently deep tank (D) filled with water. To avoid sloshing, a $3 \mathrm{~cm}$ thick flat plate of Plexiglas (E) is placed on top of the tank. The frame and tray are driven with a continuously controllable frequency $f(\operatorname{period} T)$ and amplitude $a$ by an ICME AC motor (F). The sides of the tray consist of $2 \mathrm{~cm}$ high straight boundaries, while the ends are triangular wedges with a slope of $15^{\circ}$ and maximum height $2 \mathrm{~cm}$ (see Fig. 2); the rational behind these "soft" boundaries will be discussed below. The "sand" consists of spherical glass beads, ranging in sizes from 250 to $350 \mu \mathrm{m}$. The thickness of the sand layer is smallest in the troughs of the ripple pattern, but always larger than $5 \mathrm{~mm}$. The whole setup is illuminated from the left side and filmed from above by a Dalsa 8 bit CAD4 CCD camera with $1024^{2}$ resolution. A trigger is mounted on the motor so that all pictures are taken at the same extremal position of the tray. For the values of the driving considered here (Fig. 1), typical ripple patterns consists of 15-20 ripple lengths. Suspension can be ignored and the maximal acceleration of the tray is well below the fluidization threshold. For appropriate values of $a$ and $f$, fairly regular ripple patterns grow from the flat bed. For example, for $a=2.8 \mathrm{~cm}$ we find ripple patterns with wavelength $4.2 \mathrm{~cm}$.

Grain motion - Once a fully developed pattern is formed, how does the number of grains in motion vary with $f$ and $a$ ? We observed that, due to irregularities in some grains, sand bed images usually display a number of very bright spots. The difference between two subsequent images is dominated by the appearance or disappearance of a number $n$ of such bright spots; we assume that $n$ is proportional to the number of grains which have moved.

To measure $n$ we proceed as follows: We start from a flat bed and obtain an equilibrated pattern by running the system for one hour, so that the driving amplitude selects the ripple wavelength, in contrast to the secondary instability experiments presented below. We then take
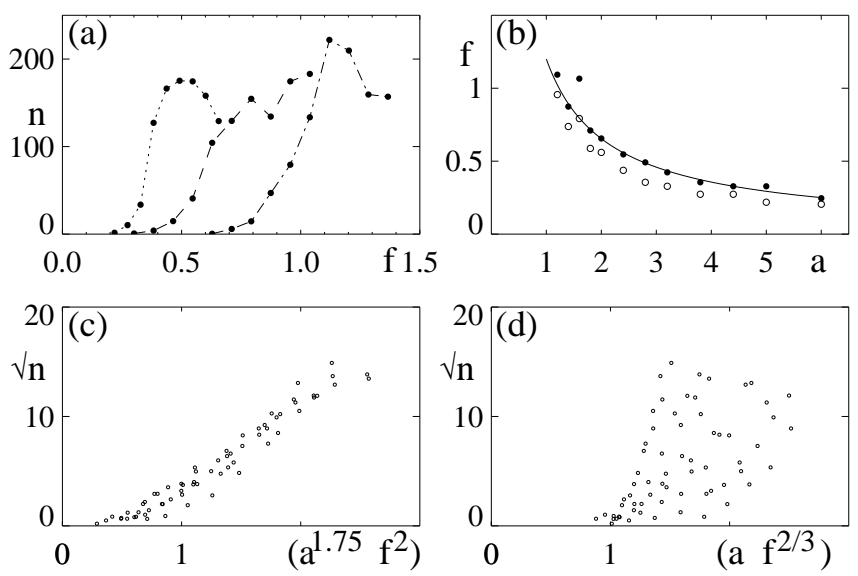

FIG. 3. (a) The average amount of moving grains $n$ as a function of the driving frequency $f$ for amplitudes 1.4 (dot-dashed), 2.4 (dashed) and 4.4 (dots). (b) Points in control parameter space where $n$ is 10 (open circles) and 50 (closed circles) compared to a curve where $a^{7 / 4} f^{2}$ is constant. (c-d) Data collapse of $\sqrt{n}$ versus the turbulent expression $a^{7 / 4} f^{2}$ and the laminar expression $A f^{2 / 3}$, with $a$ in $\mathrm{cm}$ and $f$ in $\mathrm{Hz}$ (see 10).

a series 50 images from which the average of $n$ is determined. The frequency is then lowered to a new value, the system is allowed to relax for fifteen minutes and fifty new images are recorded. This procedure is repeated for ten decreasing values of the frequency. In Fig. 3 a, three examples of the number of moving grains as function of frequency are shown. For high frequencies $n$ saturates, and the corresponding data points were discarded in the analysis presented below; this leaves us with a total of 65 data points for 10 different values of $a$.

We wish to obtain a relation between $a$ and $f$ where $n$ is constant. It was previously suggested that the maximum non-dimensional shear stress on a flat bed, $\sigma_{m}$ would be a good measure of the forcing [3, 1, 10]. If the boundary layer were laminar one would obtain that $\sigma_{m} \propto a f^{2 / 3}$ from the solution to Stokes second problem [11], while for turbulent flow a semi-empirical relation $\sigma_{m} \propto a^{1.75} f^{2}$ would be appropriate 12. In Fig. 3c we have plotted $\sqrt{n}$ versus the turbulent expression $a^{1.75} f^{2}$ and obtain a fairly good linear relation, while the correlation between $n$ and the laminar expression $a f^{2 / 3}$ is very weak (Fig. 3 d d).

Secondary instabilities - So far we have described patterns with a wavelength that is selected by the driving amplitude. Now we ask what happens when a perfectly regular pattern is suddenly subjected to changes in the driving parameters $a$ or $f$.

Obtaining a completely regular pattern is not entirely trivial. Freely grown patterns contain defects, which may be annihilated only after long times (on the order of days), although other distortions of the pattern may then appear. These are partially driven by a small drift of the pattern, which has a velocity on the order of one 

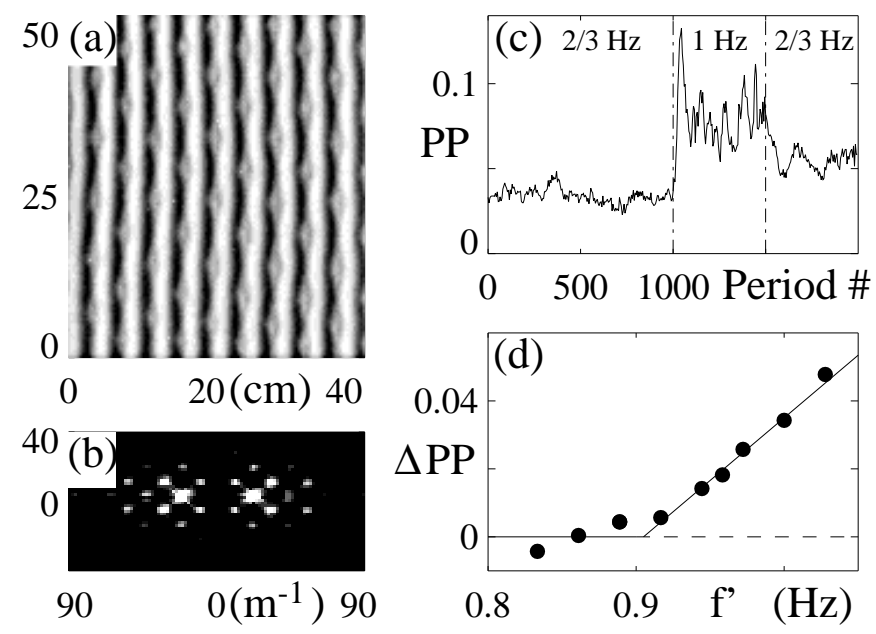

FIG. 4. (a) Central section $(43 \times 53 \mathrm{~cm})$ of a pearling state obtained by subjecting a pattern with wavelength $4.2 \mathrm{~cm}$ to a driving of $a=2.8 \mathrm{~cm}$ and $f=1 \mathrm{~Hz}$. (b) Corresponding power spectrum (range $188 \times 94 \mathrm{~cm}^{-1}$ ) (c) Time series of $P P$ when $f$ is changed from $2 / 3 \mathrm{~Hz}$ to $f^{\prime}=1 \mathrm{~Hz}$ and back again. (d) The order parameter $\triangle P P$ as a function of the frequency $f^{\prime}$. For details see text.

ripple wavelength per $10^{4}$ oscillations [13]. The deformations due to the drift are partly eliminated by using the slanted boundaries, allowing ripples to "drift out" of the system (leading to a small loss of sand at the edge of the plate). To obtain completely regular initial conditions, we have adopted the following procedure: First a flat bed is obtained by fluidizing the sand during a short period of strong oscillations of the plate. Then a regular pattern is imprinted into the sand by pressing down a frame with parallel equally spaced metal ridges. Small irregularities are then eliminated by a few $(\approx 10)$ oscillations of the plate.

By making large changes in $a$ or $f$ it is relatively simple to get regular secondary instability patterns as shown in Fig. 1 and 5 . A study of the precise nature of these transitions involves runs performed at parameters close to the instability boundaries, and here the time scale for the development of instabilities diverges. As a result, the "ideal" dynamics may get hidden behind experimental artifacts. By introducing appropriate order parameters we will below precisely characterize both the pearling and the bulging instability.

Pearling - When the driving frequency is increased beyond a certain critical value, we find a secondary "pearling" instability (Fig. (1). Here, the crests of the initial ripples remain essentially undisturbed, but in their troughs new small ripples (pearls) emerge, and the resulting pearling pattern is periodic with the pearls aligned on lines inclined by approximately 45 degrees. The pearling transition is a supercritical secondary instability. When pearls are formed, their strength quickly saturates at some well defined value, and they disappear when the frequency is lowered as shown in Fig. 田: Starting from

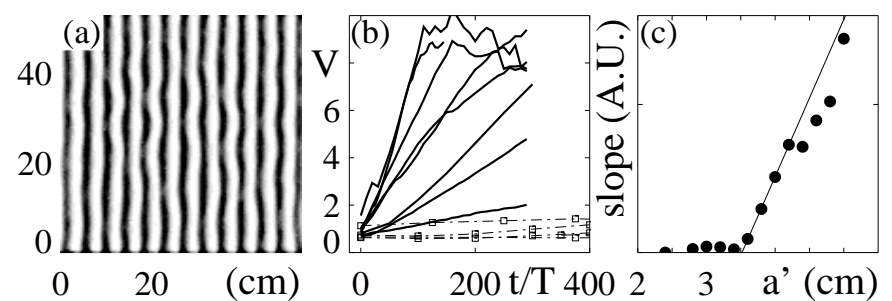

FIG. 5. (a) Central part $(53 \times 53 \mathrm{~cm})$ of a bulging pattern, $525 \mathrm{~T}$ after the driving amplitude is changed to $4 \mathrm{~cm}$ $(f=0.41)$. (b) The growth of deviations of a straight ripple pattern, quantified by the variance $V$ for $f=0.55 \mathrm{~Hz}$ and a range of amplitudes. The thin curves with symbols correspond to amplitudes 2.4, 2.8, 3.0, 3.2 and $3.4 \mathrm{~cm}$ (below the bulging instability), while the thick curves correspond to $3.6,3.8,4.0,4.2,4.4,4.6,4.8$ and $5.0 \mathrm{~cm}$ (above the bulging instability). (c) The maximum slope of $V$ (for times up to 300T) as a function of $a$ shows a sharp transition for $a \approx 3.55 \mathrm{~cm}$.

an imprinted perfect ripple pattern with wavelength 4.2 $\mathrm{cm}$ (corresponding to an amplitude $a=2.8 \mathrm{~cm}$ ), the system was driven at a low frequency of $0.67 \mathrm{~Hz}$. After 1000 oscillations, the frequency was suddenly increased to $f^{\prime}$, kept there for 500 oscillations and then again lowered to the initial frequency.

To characterize the strength of the pearls, we have measured $P P$, the total intensity in the primary satellite peaks of the power spectrum (Fig. Mb). Due to finite size effects and noise, $P P$ is not zero for perfect patterns, and as order parameter we therefore use $\triangle P P$, the difference between mean values of $P P$ during period 1000-1500 and period $0-1000$. A plot of $\triangle P P$ as a function of the quenching frequency $f^{\prime}$ shows a a well-defined transition point, above which the order parameter increases continuously (see Fig. A 4 d). A further increase of the frequency leads to more erratic states and finally to fluidization where the ripple patterns are washed away.

Bulging: - When the driving amplitude $a$ is increased sufficiently, the regular ripple patterns become unstable to two dimensional modulations. While we cannot completely rule out that this is a long wavelength instability [7], our data strongly suggests that the wavelength of this modulation in the longitudinal direction is locked on four times the wavelength of the underlying pattern, with a similar wavelength in the transversal direction. In contrast to the pearling instability, this instability is subcritical. The bulging deformations grow until neighboring ripples become so close that they form defects, which climb and glide rapidly through the system, finally leading to a regular pattern with a larger wavelength. For sequence of pictures showing the development of the instability see [9].

Close to the instability boundary it becomes very difficult to distinguish slow drift from slow development of the instability, and we have therefore developed the following sensitive measure for the onset of the instability. Starting with a perfect pattern of wavelength $4.2 \mathrm{~cm}$, we 
suddenly shift the amplitude. To characterize the time evolution of the pattern, we have extracted the local values of the "longitudinal" ripple length $\lambda_{i}$ taken over the whole two-dimensional image. The variance $V$, defined as $\sum_{i=1}^{N}\left(\lambda_{i}-\bar{\lambda}\right)^{2} /(N-1)$, is then a simple measure for the amount of deformations in the pattern. The evolution of $V$ is shown in Fig. 5b for a variety of values of the amplitude $a$.

Even below the formation of bulges, $V$ grows slowly due to slow large scale deformations of the pattern, although the growth rate is essentially independent of the driving amplitude $a$. For $a$ above some critical value, $V$ displays a clear, surprisingly linear, increase with time. In Fig. 5ic we have plotted the maximum of $(V(t f+100)-V(t f)) / 100$ for $t$ up to period 200 . This quantity, which measures the maximum slope of $V$, clearly identifies the location of the secondary instability at $a=3.55(5) \mathrm{cm}$. In this way we can distinguish between slow expansion or contraction of the pattern and the bulging instability.

Doubling - When the amplitude $a$ is decreased sufficiently, a sub-critical doubling instability occurs. The initial phase of the development of this instability suggests that it can be captured in a one-dimensional framework. When the driving amplitude gets sufficiently small, the separation vortices that drive the sand transport do no longer reach over the through between ripples. This leads to the formation of bumps in the ripple troughs, which in turn grow out to form new ripples. Behavior similar to this has been seen in numerical studies of $1 \mathrm{D}$ vortex ripple patterns [4, 14]. For a picture of this transition see [9].

Discussion and outlook - We have characterized some of the pattern forming properties of fully developed vortex ripple patterns. By observing the number of moving grains on the ripples we have showed that the maximum shields parameter on the flat bed, calculated using the turbulent expression, is a relevant control parameter. We have shown that regular vortex ripple patterns are stable to small changes in the driving amplitude and frequency. When these changes become too large, however, the vortex ripples show a rich variety of secondary instabilities; pearling, bulging and doubling.

Vortex ripples pose many theoretical challenges. The existence of a stable band and the doubling transition have been found in a simple model of ripple patterns 《]. The bulging and pearling are, to the best of our knowledge, not present in any simple theoretical models, such as Swift-Hohenberg type models incorporating local mass conservation, left right symmetry and a finite wavelength instability (which leads to spatial derivatives of order six). We believe that the origin of the instabilities is basically hydrodynamical and related to the dynamics of the separation zones. In the doubling transition it is thus clearly seen in the 1-d experiments that the new ripples originate approximately at the reconnection point for the separation vortex. The bulging and pearling transitions are genuinely $2-\mathrm{d}$ and thus more complicated, but we speculate that the bulging transition is basically a Rayleigh-Plateau "sausage" instability of the almost cylindrical separation vortex, whereas the pearling instability might be related to the centrifugal instability of the cylinder, giving rise to transverse Taylor vortices. Obviously these ideas need considerable elaboration in view of the strong time dependent shear experienced by the separation vortex.

It is a pleasure to acknowledge discussions with M.-L. Chabanol, J. Krug, A. Stegner and E. Wesfreid.

[1] R. A. Bagnold, Proc. R. Soc. London, Ser. A 187, 1 (1946).

[2] A. Stegner and J. E. Wesfried, Phys. Rev. E, 60, R3487, (1999)

[3] P. Nielsen, J. of Geoph. Res. 86(C7), 6467 (1981).

[4] K. H. Andersen, M. L. Chabanol and M. van Hecke, Phys. Rev. E, accepted.

[5] P. Blondeaux, J. Fluid Mech. 218, 1 (1990); P. Blondeaux and G. Vittori, J. Fluid Mech. 226, 257 (1991); 239, 23, (1991).

[6] K. H. Andersen, Phys. Fluids 13, 58 (2001)

[7] M. C. Cross and P. C. Hohenberg, Rev. Mod. Phys. 65, 851 (1993) and references therein.

[8] M. A. Scherer, F. Melo and M. Marder, Phys. Fluids, 11, $58,(1999)$

[9] J. L. Hansen, M. van Hecke, A. Haaning, C. Ellegaard, K. H. Andersen, T. Bohr and T. Sams, Nature (410), 324 (2001).

[10] The Shields parameter on a flat bed is defined as $\tau /\left(g d\left(\rho_{s}-\rho_{w}\right)\right)$, where $\tau$ is the maximal shear stress on the bed, $g$ denotes gravity, $d$ is the diameter of the sand grains and $\rho_{s}$ and $\rho_{w}$ are the densities of the sand and water. For laminar flow the maximal shear stress is given by $U \sqrt{2 \pi f \nu}$ where $U$ is the maximal fluid velocity, $f$ the frequency and $\nu$ the viscosity. For turbulent conditions, $\tau$ has been fitted to a numerical solution as $0.02 \rho_{w} f U^{2}\left(a / k_{N}\right)^{-0.25}$, where $a$ is the fluid amplitude and $k_{N}$ is the Nikuradze roughness of the bed 12. The number of moving grains is related to the Shields parameter as $n=6 /\left(\pi d^{2} \mu\right)\left(\sigma-\sigma_{c}\right)$, where $\sigma_{c}$ is the threshold of motion and $\mu$ the dynamical friction coefficient.

[11] L. Landau and E. Lifshitz, Fluid Mechanics, Pergamon Press (1959).

[12] J. Fredsøe, Coastal Engineering (21), 71 (1993).

[13] When one attempts to stop the drift by inserting steep ridges at each end of the moving sand tray, slow compression and dilatation of the patterns near these boundaries occurs. For such boundaries we frequently observe formation of defects after long time, even when $a$ is chosen such as to accommodate the imprinted pattern best.

[14] K. H. Andersen, Ph.D.-thesis, Niels Bohr Institute (1999), http://www.nbi.dk/ kenand/Thesis.htm 